\title{
The analgesic efficacy of the transversalis fascia plane block in iliac crest bone graft harvesting: a randomized controlled trial
}

\author{
Nicholas D. Black ${ }^{1}$, Laith Malhas ${ }^{2}$, Rongyu Jin ${ }^{1}$, Anuj Bhatia ${ }^{1}$, \\ Vincent W. S. Chan ${ }^{1}$, and Ki Jinn Chin ${ }^{1}$ \\ Department of Anesthesia, ${ }^{1}$ Toronto Western Hospital, University of Toronto, Toronto, ON, Canada, ${ }^{2}$ University \\ Hospitals Coventry and Warwickshire NHS Trust, Coventry, UK
}

\begin{abstract}
Background: Iliac crest bone graft (ICBG) harvesting is associated with significant perioperative pain and opioid consumption. This randomized controlled trial sought to determine if the transversalis fascia plane (TFP) block provides effective analgesia for anterior ICBG harvesting.

Methods: Fifty patients undergoing wrist fusion surgery with anterior ICBG harvesting were randomized to receive a TFP block with either $20 \mathrm{ml}$ of $0.5 \%$ ropivacaine or $5 \%$ dextrose. Patients additionally received a brachial plexus block for primary surgical-site anesthesia and either a general or spinal anesthetic depending on patient preference. Primary outcomes of interest were perioperative opioid consumption (measured as intravenous morphine equivalents [IME]), pain intensity at the ICBG harvest site for up to $48 \mathrm{~h}$ postoperatively, and the incidence of persistent postoperative pain at 6 and 12 months after surgery.

Results: The TFP group used less opioid in the post-anesthetic care unit (PACU) (median 0 vs. $2.5 \mathrm{mg}$ IME, $\mathrm{P}=0.01$ ) and in the first $8 \mathrm{~h}$ following PACU discharge (median 2.5 vs. $13.0 \mathrm{mg}$ IME, $\mathrm{P}=0.02$ ). The patients who received a TFP block also had lower pain scores in PACU (median 0 vs. 4.0 out of $10, \mathrm{P}<0.001$ ). Although opioid consumption and pain scores were lower in the TFP group at later timepoints, this difference was not statistically significant. Persistent pain at the ICBG site was reported in only $4.3 \%$ and $6.5 \%$ of all patients at 6 and 12 months, respectively.

Conclusions: The TFP block provides effective early analgesia for anterior ICBG harvesting. The incidence of persistent postoperative pain was low.
\end{abstract}

Keywords: Anesthesia; Conduction; Fascia; Local anesthesia; Nerve block.

Corresponding author: Ki Jinn Chin, MBBS (Hons), MMed, FRCPC

Department of Anesthesia, Toronto Western Hospital, University of Toronto, 399 Bathurst Street, Toronto, Ontario M5T 2S8, Canada

Tel: +1-416-603-5118, Fax: +1-416-603-6494, Email: gasgenie@gmail.com

ORCID: https://orcid.org/0000-0002-8339-3764

This work has previously been presented in part at the 38th Annual Regional Anesthesia and Acute Pain Medicine Meeting, May 2013, The Westin Boston Waterfront, Boston, MA, USA.

Received: December 11, 2018. Revised: January 19, 2019 (1st); February 20, 2019 (2nd); March 6, 2019 (3rd). Accepted: March 12, 2019.

Korean J Anesthesiol 2019 August 72(4): 336-343

https://doi.org/10.4097/kja.d.18.00352

(c) This is an open-access article distributed under the terms of the Creative Commons Attribution Non-Commercial License (http://creativecommons.org/ licenses/by-nc/4.0/), which permits unrestricted non-commercial use, distribution, and reproduction in any medium, provided the original work is properly cited. 


\section{Introduction}

Anterior iliac crest bone graft (ICBG) harvesting is associated with significant postoperative pain and over $25 \%$ of patients report that the ICBG pain is worse than the primary surgical site $[1,2]$.

Systemic opioids and surgical infiltration of local anesthesia have typically been used to provide analgesia for anterior ICBG; however, the effective duration of local anesthetic infiltration is short-lived (approximately $4 \mathrm{~h}$ ) [3,4]. Surgical placement of a wound catheter for continuous infusion or intermittent boluses of local anesthesia has also been described but there are conflicting reports of efficacy [1,5-7].

The transversalis fascia plane (TFP) block is an ultrasound-guided regional anesthetic technique that targets the T12 and L1 spinal nerves by injecting local anesthesia between the posterior aponeurotic extension of the transversus abdominis muscle and the deep investing transversalis fascia [8]. The use of this block has been described in a variety of clinical situations including anterior ICBG, inguinal herniorrhaphy, chronic post-herniorraphy inguinal pain, and cesarean section [9-13].

We conducted a randomized controlled trial to evaluate the efficacy of the TFP block in managing pain from the anterior ICBG site. This was done by assessing pain scores at the iliac crest and perioperative opioid consumption for patients undergoing elective forearm or wrist surgery requiring an ICBG. Patients were followed up postoperatively for 12 months to examine the incidence and severity of chronic pain at the iliac crest.

\section{Materials and Methods}

This double-blinded, randomized controlled trial was approved by the Research Ethics Board at University Hospital Network, Toronto, and prospectively registered at ClinicalTrials.org (NCT01133730).

Patients were identified from the surgical booking schedule. The inclusion criteria were elective upper limb surgery requiring ICBG, American Society of Anesthesiologists physical status IIII, 18-85 years old, and a weight greater than $50 \mathrm{~kg}$. Patients were excluded if they had a contraindication to regional anesthesia, were pregnant, had a history of long-term opioid use or a chronic pain disorder, had a history of drug or alcohol abuse, had significant psychiatric illness, had a history of severe pelvic or hip pain, or if they had an allergy to the study medications. Written informed consent was obtained prior to study inclusion.

Study participants were brought to a dedicated block room where intravenous access was obtained and routine monitors (electrocardiogram, non-invasive blood pressure, and pulse oximetry) were applied. Patients received 1-2 mg intravenous (IV) midazolam prior to the performance of an ultrasound-guided brachial plexus block. The choice of the block for the primary operative site was left to the discretion of the attending block room anesthesiologist. All brachial plexus blocks were performed with $30-40 \mathrm{ml}$ of a $1: 1$ mixture of $2 \%$ lidocaine and $0.5 \%$ bupivacaine with $5 \mu \mathrm{g} / \mathrm{ml}$ epinephrine.

Patients were randomized to receive an ultrasound guided

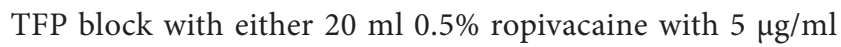
epinephrine or a placebo injection with $20 \mathrm{ml} 5 \%$ dextrose solution. Randomization was performed by a research assistant not otherwise involved in the study, utilizing a computer-generated block randomization schedule. The outcome of randomization was communicated to an anesthesia assistant who prepared the study medication in an unlabeled syringe and assisted with the performance of the block, but thereafter had no further part in the study. The patient, anesthesiologist performing the block, surgeon, anesthesiologist responsible for intraoperative care, and the personnel responsible for data collection were blinded to study group allocation.

The TFP injections were performed in the lateral position as previously described $[9,14]$ using either a linear-array high frequency $(7-12 \mathrm{MHz})$ ultrasound probe or a curved-array low-frequency $(2-5 \mathrm{MHz})$ probe in patients with truncal obesity. All TFP injections were performed by an anesthesiologist who had performed at least 10 successful TFP blocks. A 22-gauge, 80 $\mathrm{mm}$ block needle was inserted and advanced until the tip was seen to lie just deep to the transversalis fascia (Fig. 1). A $1 \mathrm{ml}$ test injection was performed to ensure correct needle placement before the remainder of the study solution was injected.

Patients then received either general anesthesia (GA) or spinal anesthesia and conscious sedation. This was decided based on patient preference and at the discretion of the attending anesthesiologist. The spinal anesthesia group received $3 \mathrm{ml}$ of intrathecal $2 \%$ mepivacaine and intraoperative sedation with an IV propofol infusion at $25-75 \mu \mathrm{g} / \mathrm{kg} / \mathrm{min}$ and IV boluses of midazolam $0.5-1.0 \mathrm{mg}$ or fentanyl $25-50 \mu \mathrm{g}$ as required. In the general anesthesia group, airway management was with either endotracheal intubation or laryngeal mask airway insertion and anesthesia was maintained with sevoflurane or desflurane at a minimum alveolar concentration of 0.8-1.4. IV boluses of fentanyl 25-50 $\mu \mathrm{g}$ were administered for analgesia at the discretion of the attending anesthesiologist.

Postoperatively, patients were transferred to the post-anesthetic care unit (PACU) where the pain scores at the site of the ICBG harvest site were documented using an 11-point numerical rating score (NRS, 0-10) and additional analgesia was administered as required by the PACU nursing staff. In accordance with the usual practice at our institution, IV fentanyl was initially administered, followed by IV morphine or hydromorphone, and oral oxycodone as necessary. A blinded research assistant assessed the extent of sensory loss to pinprick over the anterior 
abdomen in the PACU. Patients were discharged from the PACU once the routine criteria were met and received $1 \mathrm{~g}$ oral acetaminophen every $6 \mathrm{~h}$, unless contraindicated. Those admitted to the hospital overnight were prescribed IV patient-controlled analgesia (morphine 1-2 mg or hydromorphone $0.2-0.4 \mathrm{mg}$ every $5 \mathrm{~min}$ as required, lockout interval $5 \mathrm{~min}$, no basal infusion) to manage pain from the ICBG harvest site as well as the expected pain from the wrist once the effect of the brachial plexus block had worn off. Upon discharge from the hospital, all patients were instructed to take oral oxycodone $5-10 \mathrm{mg}$ or hydromorphone 2-4 mg every 3-4 h as required.

Once discharged from the PACU, each patient was asked to complete a diary to record the NRS pain scores at the ICBG harvest site and opioid consumption every $4 \mathrm{~h}$ for $48 \mathrm{~h}$. We also recorded the block duration, defined as the time from the completion of the block performance to the time of onset of increased pain at the ICBG harvest site.

Patients were asked to note the frequency of opioid-related adverse events during the first $48 \mathrm{~h}$ postoperatively on a 5-point scale ( 0 = never, 1 = rarely, 2 = occasionally, 3 = frequently, and 4 = almost constantly).

This information was gathered by a blinded research assistant. A telephone follow-up was also performed at 6 and 12 months postoperatively to assess the incidence of chronic pain at the ICBG harvest site using the Short Form McGill pain questionnaire [15] and the impact of pain on the quality of life using the SF-36v2 ${ }^{\mathrm{TM}}$ Health Survey [16].

The primary outcome was total opioid consumption in the first $24 \mathrm{~h}$; for ease of comparison all opioids administered were converted to milligram IV morphine equivalents (mg IME).

We assumed that the 24-h opioid consumption in this cohort of patients would be approximately $45 \mathrm{mg}$ IME based on a pre-

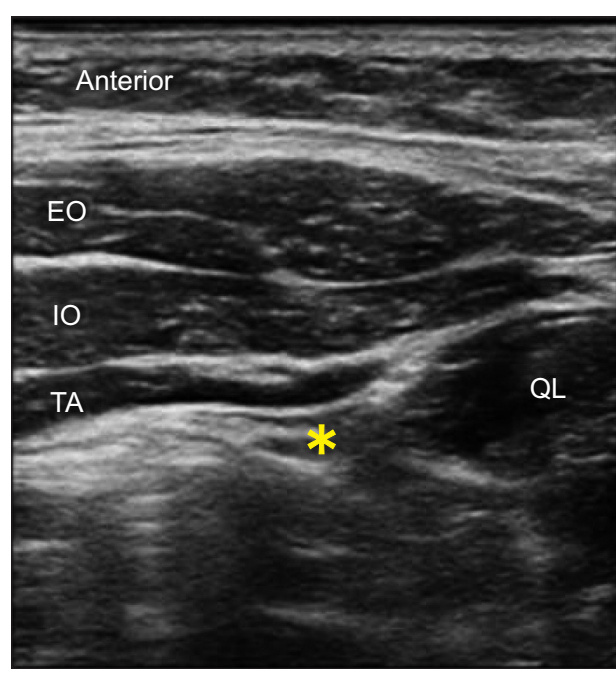

Pre-injection

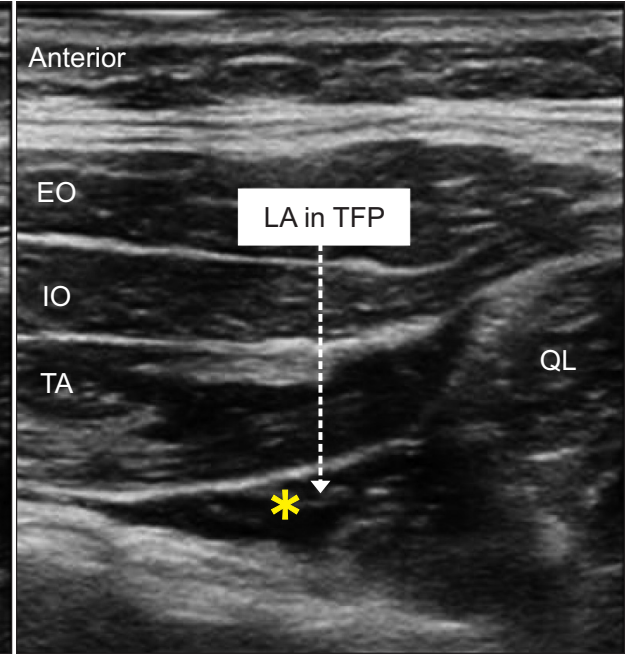

Post-injection
Fig. 1. Pre-injection and post-injection sonograms of the TFP block. The local anesthetic $\left({ }^{*}\right)$ can be seen deep to the TA muscle and pushing the perinephric fat downwards. TFP: transversalis fascia plane, TA: transversus abdominis, EO: external oblique, IO: internal oblique, QL: quadratus lumborum, and LA: local anesthetic. Reproduced with permission from Ultrasound for Regional Anesthesia (USRA; Available from http://www. usra.ca).

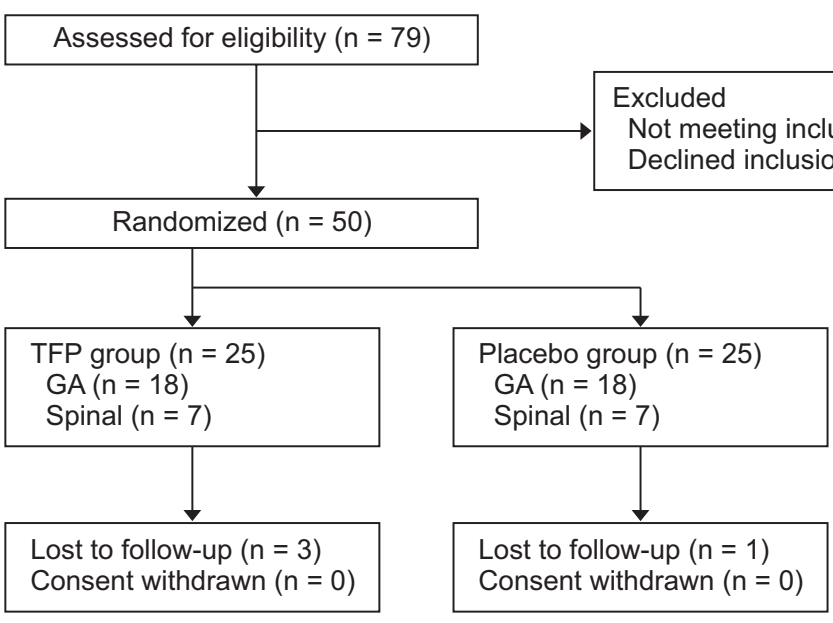

338
Fig. 2. Flow chart outlining patient inclusion. GA: general anesthesia. 
vious retrospective pilot study at our institution [9]. Assuming a power of $80 \%$ and an alpha level of 0.05 , with a standard deviation of $20 \mathrm{mg}$ IME, the study sample size was set at 28 patients per group. Statistical analysis was performed using SPSS 17.0 (SPSS Inc., USA). Continuous data were tested for normality using the Shapiro-Wilk W statistic; parametric data are reported as mean \pm standard deviation and were analyzed using the Student's $t$-test; non-parametric data is reported as median (interquartile range) and were analyzed using the Mann-Whitney $U$ test. A P value of 0.05 was chosen as the significance level.

\section{Results}

The recruitment was hindered by a change in the clinical workload of the surgeon involved and thus it was decided to

Table 1. Demographic Information of Study Participants

\begin{tabular}{llccc}
\hline \multicolumn{1}{c}{ Demographic } & & TFP & Placebo & P value \\
\hline Age $(\mathrm{yr})$ & & $45.0(16.3)$ & $48.2(12.9)$ & 0.5 \\
Sex $(\mathrm{n})$ & $\mathrm{M}$ & $60.0 \%(15)$ & $72.0 \%(18)$ & 0.6 \\
& $\mathrm{~F}$ & $40.0 \%(10)$ & $28.0 \%(7)$ & \\
BMI $\left(\mathrm{kg} / \mathrm{m}^{2}\right)$ & & $26.1(4.0)$ & $27.2(5.9)$ & 0.6 \\
Mode of & Spinal & $2.1 \%(7)$ & $28.0 \%(7)$ & 1.0 \\
anesthesia \% (n) & General & $72.0 \%(18)$ & $72.0 \%(18)$ & \\
\hline
\end{tabular}

Values are presented as mean (standard deviation) and percentage (number). BMI: body mass index, TFP: transversalis fascia plane block. stop the study earlier than anticipated. Seventy-nine patients were screened for enrolment in the study, of which a total of 50 patients were recruited and randomized to receive either a TFP block or a placebo injection (Fig. 2). Thirty-six patients received general anesthesia and 14 received spinal anesthesia, with equal numbers of patients in each group receiving a TFP block or a placebo injection. There were no significant differences in patient demographics between the groups (Table 1). The average TFP block performance time was $5.84 \pm 2.79 \mathrm{~min}$ and patients reported an NRS pain score of 2 [1-2] associated with the block. There were no complications related to the block performance in any of the patients.

Opioid consumption was significantly lower in the TFP group compared to the placebo group during their PACU stay (0 [0-2.5] vs. 2.5 [0-7.5] mg IME, $\mathrm{P}=0.01)$, during the overall perioperative period $(10.0$ [5.0-12.5] vs. 16.5 [10.0-25.0] $\mathrm{mg}$ IME, $\mathrm{P}=0.01)$, and for the first $8 \mathrm{~h}$ after PACU discharge $(2.5$ [0.4-13.5] vs. 13.0 [3.5-24.6] mg IME, $\mathrm{P}=0.02$ ) (Table 2). At all other time points examined, the reduction in opioid consumption seen in the TFP group did not achieve statistical significance (Table 2). The frequency of opioid-related side effects is shown in Table 3.

Pain scores at the ICBG site were significantly lower in the TFP group on admission to the PACU (0 [0-2.0] vs. 4.0 [0.5-7.0], $\mathrm{P}<0.001)$ and on the PACU discharge (0 [0-2.0] vs. $2.5[1.0-$ 4.8 ], $\mathrm{P}<0.001$ ) (Table 4). The average pain scores from 0 to $24 \mathrm{~h}$

Table 2. Opioid Consumption Measured in Milligram IV Morphine Equivalents (mg IME) at Different Time Periods

\begin{tabular}{|c|c|c|c|c|}
\hline Mode of anesthesia & Time period & TFP & Placebo & $\mathrm{P}$ value \\
\hline \multirow[t]{7}{*}{ All patients } & Intraoperative & $7.5(5-10)$ & $15(5-20.0)$ & 0.07 \\
\hline & PACU & $0(0-2.5)$ & $2.5(0-7.5)$ & $0.01^{*}$ \\
\hline & Overall perioperative & $10.0(5.0-12.5)$ & $16.5(10.0-25.0)$ & $0.01^{*}$ \\
\hline & $0-8 \mathrm{~h}$ & $2.5(0.4-13.5)$ & $13.0(3.5-24.6)$ & $0.02^{*}$ \\
\hline & $0-24 \mathrm{~h}$ & $28.0(13.5-46.1)$ & $28.0(13.9-50.5)$ & 0.85 \\
\hline & $24-48 \mathrm{~h}$ & $0(0-5.0)$ & $0(0-5.0)$ & 0.79 \\
\hline & $48-72 \mathrm{~h}$ & $0(0-1.9)$ & $0(0-0)$ & 0.42 \\
\hline \multirow[t]{7}{*}{ GA patients only } & Intraoperative & $10.0(5.0-13.8)$ & $15.0(10.0-23.1)$ & $0.02^{*}$ \\
\hline & PACU & $0(0-2.5)$ & $3.8(0-10.0)$ & $0.03^{*}$ \\
\hline & Overall perioperative & $10.0(6.9-18.1)$ & $17.5(14.4-29.4)$ & $0.004^{*}$ \\
\hline & $0-8 \mathrm{~h}$ & $4.5(0.8-14.0)$ & $14.0(5.0-25.3)$ & $0.03^{*}$ \\
\hline & $0-24 \mathrm{~h}$ & $25.0(12.5-43.0)$ & $30.8(22.9-65.9)$ & 0.25 \\
\hline & $24-48 \mathrm{~h}$ & $0(0-2.5)$ & $0(0-8.8)$ & 0.31 \\
\hline & $48-72 \mathrm{~h}$ & $0(0-3.8)$ & $0(0-0.6)$ & 0.64 \\
\hline \multirow[t]{7}{*}{ Spinal anesthesia patients only } & Intraoperative & $5.0(0-5.0)$ & $5(0-7.5)$ & 0.79 \\
\hline & PACU & $0(0-0)$ & $0(0-5.0)$ & 0.17 \\
\hline & Overall perioperative & $5.0(1.0-5.0)$ & $7.5(2.5-10.0)$ & 0.27 \\
\hline & $0-8 \mathrm{~h}$ & $1.0(0-12.0)$ & $3.0(0-16.0)$ & 0.40 \\
\hline & $0-24 \mathrm{~h}$ & $28.0(15.7-50.0)$ & $15.0(6.0-19.0)$ & 0.09 \\
\hline & $24-48 \mathrm{~h}$ & $0(0-12.5)$ & $0(0-0)$ & 0.14 \\
\hline & $48-72 \mathrm{~h}$ & $0(0-0)$ & $0(0-0)$ & 0.32 \\
\hline
\end{tabular}

Values are presented as median (interquartile range). Mann-Whitney $U$ tests were performed to assess significance. GA: general anesthesia, PACU: postoperative care unit, TFP: transversalis fascia plane block. *Indicates a statistically significant result with $\mathrm{P}<0.05$. 
and 24 to $48 \mathrm{~h}$ following PACU discharge were lower in the TFP group at other time points but this difference did not achieve statistical significance (Table 4).

Upon subgroup analysis, those patients in the TFP group who received GA used significantly less opioid intraoperatively (10.0 [5.0-13.8] vs. 15.0 [10.0-23.1] mg IME, $\mathrm{P}=0.02)$, in the PACU (0 [0-2.5] vs. 3.75 [0-10.0] mg IME, $\mathrm{P}=0.03)$, and overall during the perioperative period (10.0 [6.9-18.1] vs. 17.5 [14.4-29.4] mg IME, $\mathrm{P}=0.004$ ) (Table 2). This reduction was also significant during the first $8 \mathrm{~h}$ after the PACU discharge (4.5 [0.8-14.0] vs. 14.0 [5.0-25.3] mg IME, $\mathrm{P}=0.02$ ) but not at any later time period. Regarding pain scores, the patients in the TFP group who underwent GA had significantly less pain on admission to the PACU (0 [0-2.0] vs. $4.5[1.8-7.0], \mathrm{P}<0.001)$ and on

Table 3. Frequency of Opioid Related Side Effects (\%)

\begin{tabular}{llccccc}
\hline \multirow{2}{*}{ Adverse effect } & \multicolumn{2}{c}{ TFP } & & \multicolumn{2}{c}{ Placebo } & \\
\cline { 2 - 3 } \cline { 5 - 6 } & $0-2$ & $3-4$ & & $0-2$ & $3-4$ & \\
\hline Nausea & 84 & 16 & 86.4 & 13.6 & 0.63 \\
Vomiting & 91.7 & 8.3 & 100 & 0 & $0.003^{*}$ \\
Constipation & 95.8 & 4.2 & 95.2 & 4.8 & 0.84 \\
Dysuria & 87.5 & 12.5 & 95.7 & 4.3 & $0.04^{*}$ \\
Concentration & 80 & 20 & 86.4 & 13.6 & 0.23 \\
Drowsiness & 68 & 32 & 69.6 & 30.4 & 0.80 \\
Dizziness & 92 & 8 & 86.4 & 13.6 & 0.20 \\
Fatigue & 64 & 36 & 56.5 & 43.5 & 028 \\
Confusion & 96 & 4 & 95.5 & 4.5 & 0.86 \\
Itchiness & 84 & 16 & 90.9 & 9.1 & 0.14 \\
Dry mouth & 64 & 36 & 60.9 & 39.1 & 0.65 \\
Headache & 92 & 8 & 95.5 & 4.5 & 0.31 \\
\hline
\end{tabular}

Frequency of side effects were reported by patients on a 5-point scale $(0=$ never, $1=$ rarely, $2=$ occasionally, $3=$ frequently and $4=$ almost constantly). TFP: transversalis fascia plane block. *Indicates a statistically significant result with $\mathrm{P}<0.05$. discharge from the PACU (0 [0-2.0] vs. 4.0 [1.5-5.5], P < 0.001).

In the subgroup of patients who underwent spinal anesthesia, there was no significant difference between the groups in opioid consumption or pain scores at any time points analyzed (Tables 2 and 4$)$.

We were able to perform postoperative sensory testing in 23 of the 25 patients in the TFP group. All patients had a sensory loss to touch in the L1 dermatome, but no higher in 65\% of the patients. In one patient the upper limit of sensory loss reached T6 with the remainder ranging from T7 to T12 (Fig. 3).

Block duration was assessed in 11 of the 25 patients in the TFP group; the remaining patients had either no pain at the ICBG harvest site or were unable to discern if and when there was any increase in pain. The mean block duration recorded was $11.7 \pm 4.9 \mathrm{~h}$.

Assessments for persistent postoperative pain were completed for all but three and four patients at 6 months and 12 months, respectively. There was no statistically significant difference in the SF-36 Physical Component Summary or Mental Component Summary scores between the groups at both time periods. At 6 months, one patient in each group reported mild pain during activity at the ICBG harvest site. At 12 months, one patient in the placebo group had mild pain and two patients (one in each group) had moderately severe pain with activity. Due to the small numbers, no further analysis was performed on the chronic pain data.

\section{Discussion}

The results of this study demonstrate that the TFP block is an effective analgesic technique in anterior ICBG harvesting. The TFP block is quick to perform, well tolerated in the minimally-sedated patient, consistently provides unilateral sensory

Table 4. Average Pain Numerical Rating Scores (NRS) at the ICBG Harvest Site

\begin{tabular}{|c|c|c|c|c|}
\hline Mode of anesthesia & Time & TFP & Placebo & $P$ value \\
\hline \multirow[t]{4}{*}{ All patients } & PACU admission & $0(0-2.0)$ & $4(0.5-7.0)$ & $<0.001^{*}$ \\
\hline & PACU discharge & $0(0-2.0)$ & $2.5(1.0-4.8)$ & $<0.001^{*}$ \\
\hline & $0-24 \mathrm{~h}$ average & $1.5(0-4.0)$ & $3.0(1.0-5.0)$ & 0.07 \\
\hline & $24-48 \mathrm{~h}$ average & $1.5(0.6-3.8)$ & $3.5(1.0-5.0)$ & 0.24 \\
\hline \multirow[t]{4}{*}{ GA patients only } & PACU admission & $0(0-2.0)$ & $4.5(1.8-7.0)$ & $<0.001^{*}$ \\
\hline & PACU discharge & $0(0-2.0)$ & $4.0(1.5-5.5)$ & $<0.001^{*}$ \\
\hline & $0-24 \mathrm{~h}$ average & $1.0(0.3-4.0)$ & $3.0(1.5-5.0)$ & 0.08 \\
\hline & $24-48 \mathrm{~h}$ average & $1.5(0.8-3.5)$ & $3(1.0-5.0)$ & 0.15 \\
\hline \multirow[t]{4}{*}{ Spinal anesthesia patients only } & PACU admission & $0(0-0)$ & $0(0-4.0)$ & 0.53 \\
\hline & PACU discharge & $0(0-2.0)$ & $1.0(0-2.0)$ & 0.30 \\
\hline & $0-24 \mathrm{~h}$ average & $2.0(0-4.0)$ & $2.8(0.8-5.0)$ & 0.51 \\
\hline & $24-48 \mathrm{~h}$ average & $1.0(0-4.0)$ & $4.0(1.0-4.0)$ & 0.36 \\
\hline
\end{tabular}

$\overline{\text { Values are presented as median (interquartile range). The Reduction in pain scores only achieved statistical significance in the post-anesthetic care }}$ unit (PACU). Mann-Whitney $U$ tests were performed to assess significance. GA: general anesthesia, TFP: transversalis fascia plane block. *Indicates a statistically significant result with $\mathrm{P}<0.05$. 


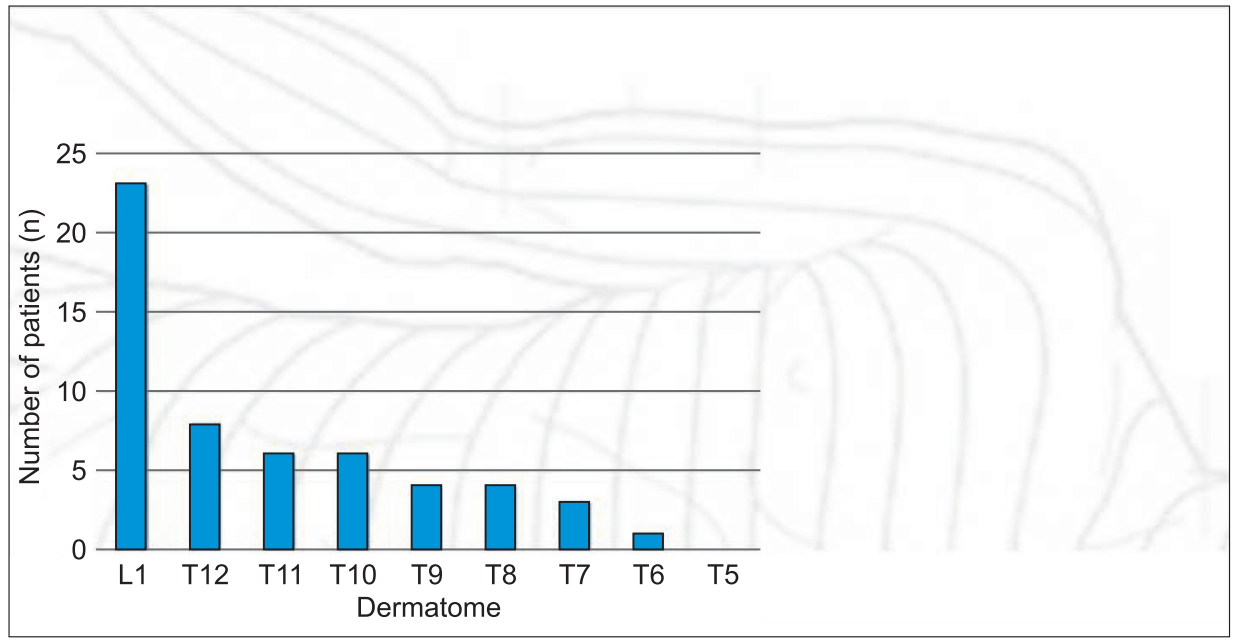

Fig. 3. The numbers of patients with sensory loss to touch at each dermatomal level. All patients had a block at L1; however, the block was limited to this dermatome in the majority (65\%). One patient had a block that extended to T6. blockade of the L1 dermatome, and provides analgesia for almost $12 \mathrm{~h}$ on an average.

Pain at the ICBG harvest site was generally of low intensity and, despite a trend towards lower pain scores in the TFP group up to $48 \mathrm{~h}$ postoperatively, there was no statistically significant difference between the groups after the PACU discharge. The lack of difference in the pain scores may be attributed to effective systemic analgesia in the group receiving a placebo injection and is reflected in the significantly higher opioid consumption that was observed in these patients during the first $8 \mathrm{~h}$ following PACU discharge. Thereafter, there was no significant difference in opioid consumption between the groups. This may be attributed to the offset of the brachial plexus block and the onset of pain at the primary surgical site at the wrist, which would have required opioid analgesia for effective management.

The reported incidence of persistent postoperative pain following ICBG harvesting is variable; in one study, the iliac crest pain was present 2 years after surgery in $21.1 \%$ of the patients who had had an ICBG for spine surgery compared to only $6.2 \%$ for non-spine surgery [17]. Spine surgery usually involves the harvest of larger segments of the bone as well as the use of a posterior iliac crest site versus an anterior one. The intensity of acute postoperative pain is much greater, which in turn is a significant risk factor for the development of persistent postoperative pain [18]. The low incidence of chronic pain observed in the current study is thus not surprising, but it does prevent us from determining if the TFP block may contribute to a reduction in the incidence of this complication.

One of the major limitations in our study was the fact that some patients in this study received spinal anesthesia and others general anesthesia to provide surgical anesthesia for the ICBG harvest site. Unfortunately, at the time the study was conducted, this was routine institutional practice and the exclusion of either modality would have significantly hampered study re- cruitment. The use of spinal anesthesia would have masked any intraoperative benefit of the TFP block, and this confounding was further exacerbated by the common practice in our institution to administer incremental doses of fentanyl for conscious sedation during regional anesthesia. Outcome assessment in the PACU was impacted to a lesser extent as patients were discharged only after the sensory level had regressed to the L3 dermatome or lower. We attempted to address these limitations with a subgroup analysis by mode of anesthesia. Results in the general anesthesia subgroup were similar to the overall analysis in that patients who received a TFP block used significantly less opioid at all time points up to $8 \mathrm{~h}$ following PACU discharge and had lower pain scores in PACU. In addition, the TFP block also reduced intraoperative opioid consumption in the GA subgroup. In contrast, the reductions in the smaller spinal anesthesia subgroup failed to reach statistical significance at any time point. This however may represent a type 2 error given the small sample size of the subgroup. Another related limitation of our study was the failure to reach the targeted sample size due to difficulties with recruitment. This may have contributed to the lack of a statistically significant difference in the primary outcome of 24-h opioid consumption.

Transversus abdominis plane (TAP) blocks [13,19] and transmuscular quadratus lumborum (TQL) [20] blocks have been used to provide analgesia for ICBG. One concern in using TAP blocks for ICBG is that they may not reliably cover the L1 dermatome [21,22]. A study by Lee et al. [22] showed that an ultrasound-guided posterior TAP block will produce sensory loss in the L1 dermatome less than $50 \%$ of the time. The TQL block may be more consistent and a case series of five patients undergoing ICBG by Sondekoppam et al. [20] showed that there was sensory loss in the L1 dermatome in all cases. In the present study, the TFP block provided sensory loss in the L1 dermatome in all patients and, unlike the TQL block, does not require access 
to the back or use of a curvilinear probe in the majority of patients.

We did not note any complications of the TFP block or placebo injection in the current study, although the potential safety concerns are similar to that of other abdominal wall blocks, including visceral injury, vascular injury, and local anesthetic systemic toxicity [23]. However, there is one report of quadriceps weakness following a TFP block, which was believed to have been caused by the proximal spread of local anesthetic to the lumbar plexus [24]. Lower limb weakness has also been described following quadratus lumborum [25] and TAP [26,27] blocks, suggesting that vigilance is warranted when mobilizing patients.

In summary, an ultrasound-guided TFP block is a relatively simple regional anesthetic technique that reliably blocks the L1 dermatome and can provide early postoperative analgesia in patients undergoing anterior ICBG harvesting.

\section{Acknowledgments}

Dr. Danielle Factor and Sabeeh Alvi for their contributions to the development of the study and assistance with data analysis. Dr. Brent Graham for his advice on study design and assistance with study recruitment.

\section{Funding Statement}

Dr. Ki Jinn Chin was supported by a Merit Award from the Department of Anesthesia, University of Toronto.

\section{Conflicts of Interest}

Dr. Vincent Chan has received honorarium from Aspen Pharma, BBraun, Smiths Medical, and SonoSite.

\section{Author Contributions}

Nicholas D. Black (Formal analysis; Investigation; Writing-original draft; Writing-review \& editing)

Laith Malhas (Investigation; Writing-original draft; Writingreview \& editing)

Rongyu Jin (Data curation; Writing-original draft; Writingreview \& editing)

Anuj Bhatia (Conceptualization; Investigation; Methodology; Writing-review \& editing)

Vincent W. S. Chan (Conceptualization; Investigation; Methodology; Writing-review \& editing)

Ki Jinn Chin (Conceptualization; Data curation; Investigation; Methodology; Writing-original draft; Writing-review \& editing)

\section{ORCID}

Nicholas D. Black, https://orcid.org/0000-0001-8580-3897

Laith Malhas, https://orcid.org/0000-0003-0765-3373

Rongyu Jin, https://orcid.org/0000-0003-1805-283X

Anuj Bhatia, https://orcid.org/0000-0001-6292-8654

Vincent W. S. Chan, https://orcid.org/0000-0003-0696-708X

Ki Jinn Chin, https://orcid.org/0000-0002-8339-3764

\section{References}

1. Morgan SJ, Jeray KJ, Saliman LH, Miller HJ, Williams AE, Tanner SL, et al. Continuous infusion of local anesthetic at iliac crest bone-graft sites for postoperative pain relief. A randomized, double-blind study. J Bone Joint Surg Am 2006; 88: 2606-12.

2. DeOrio JK, Farber DC. Morbidity associated with anterior iliac crest bone grafting in foot and ankle surgery. Foot Ankle Int 2005; 26: 14751.

3. Todd BD, Reed SC. The use of bupivacaine to relieve pain at iliac graft donor sites. Int Orthop 1991; 15: 53-5.

4. Cowan N, Young J, Murphy D, Bladen C. Double-blind, randomized, controlled trial of local anesthetic use for iliac crest donor site pain. J Neurosci Nurs 2002; 34: 205-10.

5. Blumenthal S, Dullenkopf A, Rentsch K, Borgeat A. Continuous infusion of ropivacaine for pain relief after iliac crest bone grafting for shoulder surgery. Anesthesiology 2005; 102: 392-7.

6. Singh K, Phillips FM, Kuo E, Campbell M. A prospective, randomized, double-blind study of the efficacy of postoperative continuous local anesthetic infusion at the iliac crest bone graft site after posterior spinal arthrodesis: a minimum of 4-year follow-up. Spine (Phila Pa 1976) 2007; 32: 2790-6.

7. Brull SJ, Lieponis JV, Murphy MJ, Garcia R, Silverman DG. Acute and long-term benefits of iliac crest donor site perfusion with local anesthetics. Anesth Analg 1992; 74: 145-7.

8. Hebbard PD. Transversalis fascia plane block, a novel ultrasound-guided abdominal wall nerve block. Can J Anaesth 2009; 56: 618-20.

9. Chin KJ, Chan V, Hebbard P, Tan JS, Harris M, Factor D. Ultrasound-guided transversalis fascia plane block provides analgesia for anterior iliac crest bone graft harvesting. Can J Anaesth 2012; 59: 122-3.

10. López-González JM, López-Álvarez S, Jiménez Gómez BM, Areán González I, Illodo Miramontes G, Padín Barreiro L. Ultrasound-guided transversalis fascia plane block versus anterior transversus abdominis plane block in outpatient inguinal hernia repair. Rev Esp Anestesiol 
Reanim 2016; 63: 498-504.

11. Choudhary J, Mishra AK, Jadhav R. Transversalis fascia plane block for the treatment of chronicpostherniorrhaphy inguinal pain: a case report. A A Pract 2018; 11: 57-9.

12. Tulgar S, Serifsoy TE. Transversalis fascia plane block provides effective postoperative analgesia for cesarean section: New indication for known block. J Clin Anesth 2018; 48: 13-4.

13. Punekar IR, Koltz PF, Smith DI, Tran NH, Chibber AK, Sbitany H, et al. The evolution of iliac bone graft donor site analgesia in cleft patients: transversus abdominis plane block is safe and efficacious. Ann Plast Surg 2018; 81: 441-3.

14. Chin KJ. Transversalis fascia plane block [Internet]. USRA: Advancing the Science of Ultrasound Guided Regional Anesthesia and Pain Medicine [cited 2018 Nov 13]. Available from http://www.usra.ca/regional-anesthesia/specificblocks/trunk/tfpblock.php.

15. Melzack R. The short-form McGill Pain Questionnaire. Pain 1987; 30: 191-7.

16. Maruish ME. User's manual for the SF-36v2 health survey. Quality Metric Incorporated, 2011. Available from https://books.google.ca/ books?id=a0vYnQEACAAJ.

17. Goulet JA, Senunas LE, DeSilva GL, Greenfield ML. Autogenous iliac crest bone graft. Complications and functional assessment. Clin Orthop Relat Res 1997; (339): 76-81.

18. Richebé P, Capdevila X, Rivat C. Persistent postsurgical pain: pathophysiology and preventative pharmacologic considerations. Anesthesiology 2018; 129: 590-607.

19. Chiono J, Bernard N, Bringuier S, Biboulet P, Choquet O, Morau D, et al. The ultrasound-guided transversus abdominis plane block for anterior iliac crest bone graft postoperative pain relief: a prospective descriptive study. Reg Anesth Pain Med 2010; 35: 520-4.

20. Sondekoppam RV, Ip V, Johnston DF, Uppal V, Johnson M, Ganapathy S, et al. Ultrasound-guided lateral-medial transmuscular quadratus lumborum block for analgesia following anterior iliac crest bone graft harvesting: a clinical and anatomical study. Can J Anaesth 2018; 65: 178-87.

21. Børglum J, Jensen K, Christensen AF, Hoegberg LC, Johansen SS, Lönnqvist PA, et al. Distribution patterns, dermatomal anesthesia, and ropivacaine serum concentrations after bilateral dual transversus abdominis plane block. Reg Anesth Pain Med 2012; 37: 294-301.

22. Lee TH, Barrington MJ, Tran TM, Wong D, Hebbard PD. Comparison of extent of sensory block following posterior and subcostal approaches to ultrasound-guided transversus abdominis plane block. Anaesth Intensive Care 2010; 38: 452-60.

23. Chin KJ, McDonnell JG, Carvalho B, Sharkey A, Pawa A, Gadsden J. Essentials of our current understanding: abdominal wall blocks. Reg Anesth Pain Med 2017; 42: 133-83.

24. Lee S, Goetz T, Gharapetian A. Unanticipated motor weakness with ultrasound-guided transversalis fascia plane block. A A Case Rep 2015; 5: 124-5.

25. Ueshima H, Hiroshi O. Incidence of lower-extremity muscle weakness after quadratus lumborum block. J Clin Anesth 2018; $44: 104$.

26. Manatakis DK, Stamos N, Agalianos C, Karvelis MA, Gkiaourakis M, Davides D. Transient femoral nerve palsy complicating "blind" transversus abdominis plane block. Case Rep Anesthesiol 2013; 2013: 874215.

27. Walker G. Transversus abdominis plane block: a note of caution! Br J Anaesth 2010; 104: 265. 